\title{
Sparse Scanning Electron Microscopy and Deep Learning for Imaging and Segmentation of Neuron Structures
}

\author{
Tim Dahmen ${ }^{1, *}$, Pavel Potocek ${ }^{2}$, Patrick Trampert ${ }^{1,3}$, Maurice Peemen ${ }^{2}$ and Remco Schoenmakers ${ }^{2}$ \\ 1. German Research Center for Artificial Intelligence (DFKI), Saarbrücken, Germany. \\ 2. Thermo Fischer Scientfic, Eindhoven, The Netherlands. \\ 3. Saarland University, Saarbrücken, Germany. \\ * Corresponding author: Tim.Dahmen@dfki.de
}

Sparse Scanning Electron Microscopy can be used in combination with Inpainting algorithms to reduce acquisition time and electron dose. In many situations, this approach leads to a higher image quality compared to images obtained by a conventional raster scan acquired at the same dose per pixel [1]. But what is the definition of image quality in this context? Human observers are unavoidably biased to prefer images that look good to humans. Most modern image quality metrics contain some "perception factor" as well that links the definition of image quality to the human visual systems. However, images are increasingly processed by machine learning systems such as Deep Neural Networks and evaluated automatically. In this context, the definition of image quality should be linked to the capability of a machine learning system to extract information from the image, not the human visual system.

In this study [2] we demonstrate a workflow that acquires data by performing a sparse scan at random positions on a specimen. The sparse data is reconstructed to a full grid image by a dictionary based inpainting algorithm, implemented using high performance computing techniques on a graphics processing unit (GPU) [3]. The reconstructed data is suitable to be used for automatic semantic segmentation of neuron structures, for example using U-net shaped Convolutional Neural Network $(\mathrm{CNN})$. We found that: (1) If the network is trained from perfect data acquired from a full raster scan at high dwell time, the segmentation quality suffers from the unavoidable reconstruction results present in the inpainted images. (2) If the network is trained from reconstructed sparse data that suffers from the same type of artifact as the evaluation set images, the network "learns" to handle the artifacts in the data and the final segmentation improves. We demonstrate the procedure on two key segmentation applications in connectomics (cell membranes and mitochondria) and show that the overall segmentation quality improves notably compared to data from a conventional raster scan acquired with the same total dose per image. Alternatively, the total dwell time per pixel can be reduced by $33 \%$ while maintaining the same level of quality of the segmentation. These results demonstrate that sparse scanning and reconstruction can increase the effective data acquisition rates without sacrificing on quality for the end user segmentation application. However, the training data must be generated by the same procedure that will be used during the final imaging [4].

\section{References:}

[1] F Boughorbel et al., Microsc. Microanal. 23(S1) (2017), p. 150.

[2] P Potocek et al., IEEE International Conference on Bioinformatics and Biomedicine (2018).

[3] P Trampert et al., Microsc. Microanal. 24(S1) (2018), p. 700.

[4] The authors acknowledge funding from Thermo Fischer Scientfic, Eindhoven, The Netherlands. 
a

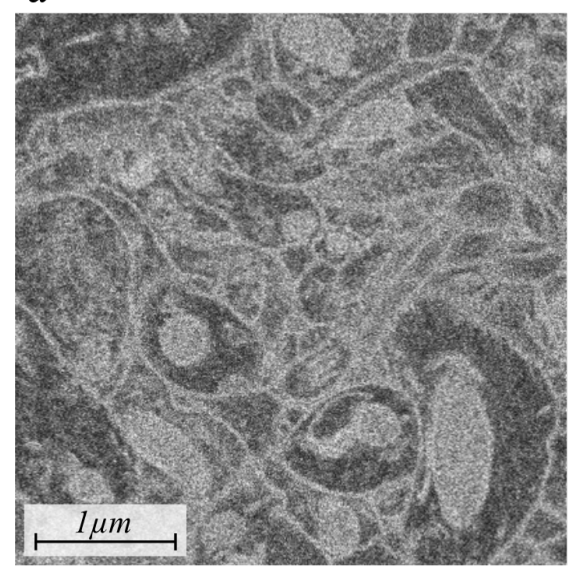

d

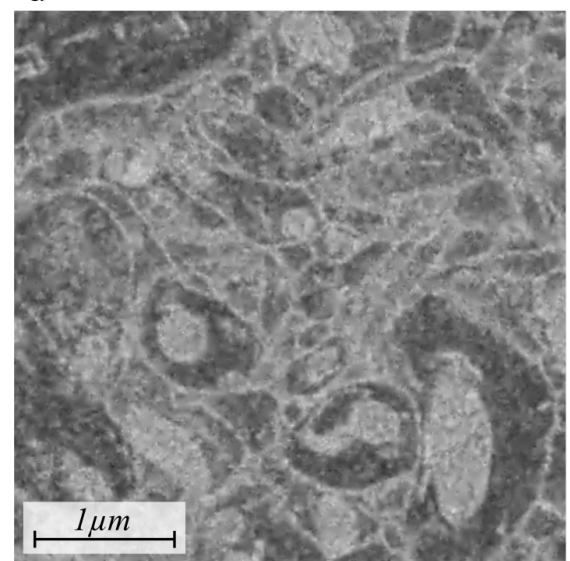

b

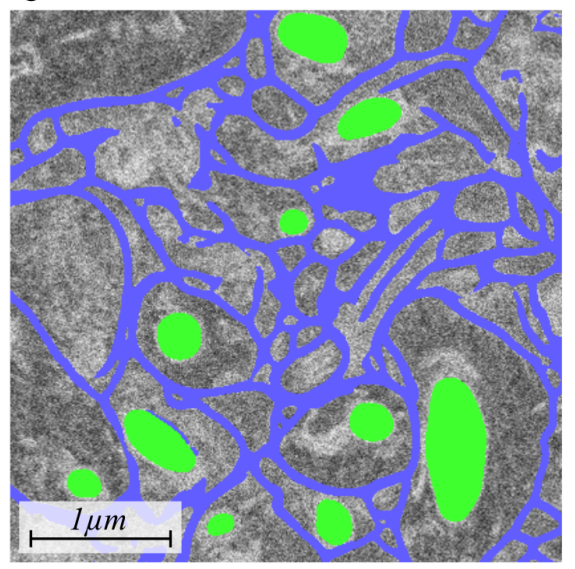

$\mathrm{e}$

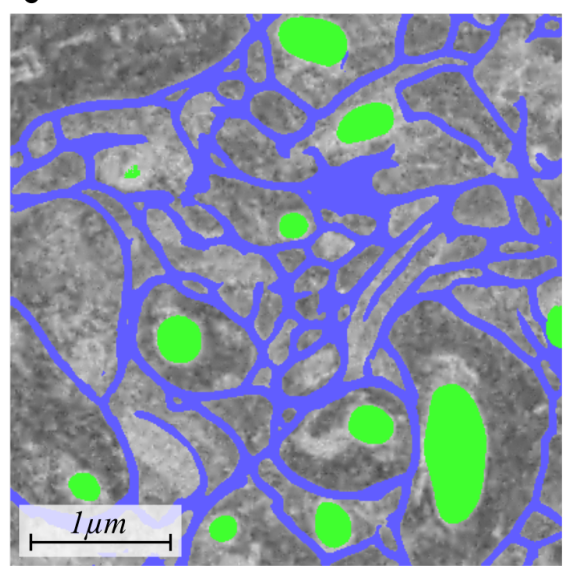

$\mathrm{c}$

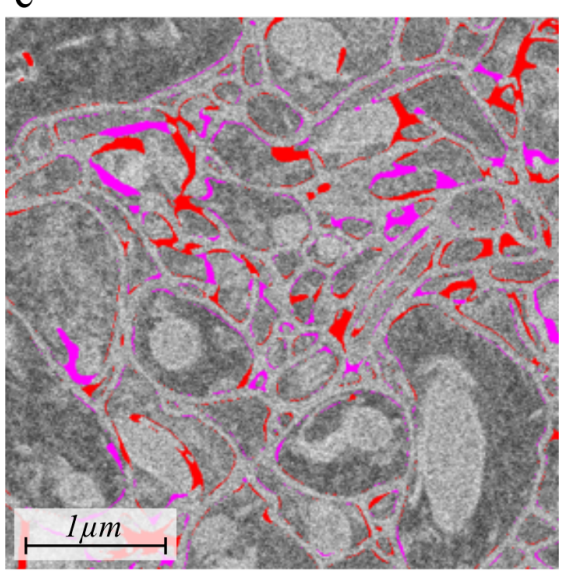

f

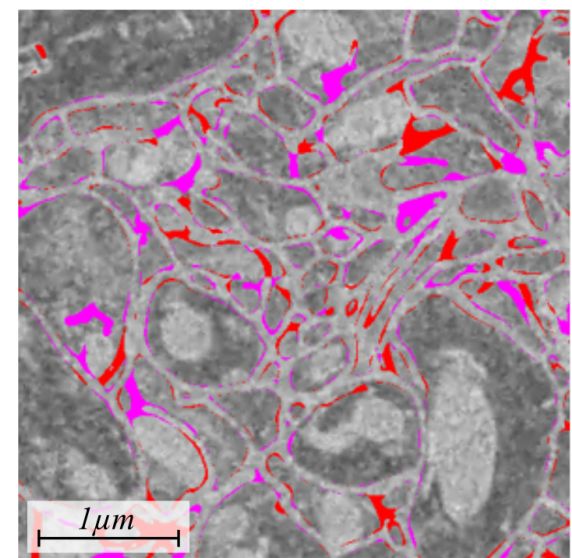

Figure 1. Results of the segmentation procedure on sparse images. The left column (a,d) shows the input images, the middle column $(\mathrm{b}, \mathrm{e})$ shows the segmentation results with mitochondria in green and membranes in blue. The right column $(\mathrm{c}, \mathrm{f})$ shows errors in the segmentation. Red pixels are false positives, pink pixels are false negatives. The first row (a-c) shows results from a full scan acquired with a pixel dwell time of $1 \mu \mathrm{s}$, The bottom tow (d-f) shows results from a sparse scan where only $20 \%$ of all pixels are acquired, also at a pixel dwell time of $3 \mu \mathrm{s}$. 